\title{
Hidrodinâmica de Líquidos Imiscíveis (Água-Óleo) em escoamentos internos: Seção Reta Circular e Placas Planas Paralelas
}

\section{Hydrodynamics of immiscible liquids (water-oil) in internal flows: cross section circular and plane parallel plates}

\author{
Artur Kimura ${ }^{1}$ \\ Carolina Valente de Oliveira ${ }^{2}$ \\ Elcio Nogueira ${ }^{3}$
}

Original

Paper

Palavras-chave:

Escoamento laminar

Dutos circulares

Placas planas

paralelas

Fluídos imísciveis

\section{Resumo:}

Em muitas operações industriais encontram-se situações em que dois líquidos devem escoar simultaneamente por um mesmo duto. Neste trabalho, apresenta-se um estudo analítico do comportamento do escoamento laminar completamente desenvolvido de fluidos imiscíveis (água-óleo) em placas planas e dutos circulares. O sistema empregado é o "Core Flow Lift", que consiste em adicionar ao fluxo de óleo na tubulação um fluido de menor viscosidade, na forma de uma capa anular externa (água), o que reduz consideravelmente a resistência do óleo ao escoamento, conseguindo um ganho na relação de potência de bombeamento. Esse processo é de fácil aplicação, baixo custo e alto rendimento, possibilitando altas vazões, e já vem sendo utilizado no transporte à longa distância em algumas plantas no exterior, com sucesso. As situações físicas de escoamento entre placas planas paralelas e dutos circulares de seção circular, além de serem muito aplicadas na indústria, são as duas geometrias mais estudadas na literatura utilizada nos Cursos de Graduação em Engenharia Mecânica. Foram desenvolvidos os perfis de velocidades de cada fluido para diferentes campos de temperatura de mistura, partindo-se dos perfis de velocidades e das tensões viscosas na interface dos fluidos, considerando contato perfeito entre ambos os meios. Demonstra-se que existe uma temperatura ideal para redução na potência de bombeamento para duto de seção reta circular e uma temperatura limitante para sistema água-óleo em placas planas paralelas.

\section{Abstract}

In many industrial operations there are situations that two fluids should flown simultaneously through the same duct. This work presents an analytical study of the laminar flown behavior fully developed by immiscible fluids (water-oil) in flat plates and circular ducts. The system employed is the "Core Flow Lift", which consists of adding a lower viscosity fluid in a form of an outer ring cover (water) to the flow of oil in the pipe. This reduces the resistance of oil to the flow, achieving a gain in the power pumping relation. This process is easy to use, it has a low cost and high performance, allowing high flow rates, and it has been used in long-distance transport in some plants abroad with success. The physical situations of flow between flat parallel plates and circular ducts of circular section are the two most studied geometries in literature used in Mechanical engineering courses, beyond being applied in industry. The
Key words:

Laminar flow

circular ducts

flat parallel plates

immiscible fluids
Recebido em $12 / 2010$

Aprovado em $12 / 2011$

Bacharel em Engenharia Mecânica - UniFOA

${ }^{2}$ Discente do 6 período de Engenharia Mecânica - UniFOA

${ }^{3}$ Professor Doutor Elcio Nogueira - Engenharia Mecânica - UniFOA 
speed profiles of each fluid to the different fields of mixture temperature were developed, starting from the speed profiles and the viscous tensions in the fluids interface, considering the perfect contact between both media. It shows the existence of an ideal temperature to the reduction of the power pumping to the duct of circular cross section and a limiting temperature to the oil-water system in flat parallel plates.

\section{Nomenclatura}

K- Gradiente de pressão

$K^{\text {th }}$ - Gradiente de pressäo do fluido interno em contato com a parede

$\frac{d p}{d z}-$ Gradiente de pressão

$\mu_{\mathrm{m}}-$ Viscosid.a.de dinâmica da. região $m$

$\mu_{1}$ - Viscosidade dinâmica da região 1

$\mu_{2}-$ Viscosidade dinâmica da região 2

$r$-Coordenada radial

$n$-Coeficiente que define a geometria do problema: $n=0$ (placa plana paralela) $n=1$ (duto circular)

$R=\left(\frac{r}{r_{2}}\right)-$ Coordenada radial admensional

$\delta=\left(\frac{r_{1}}{r_{2}}\right)-$ Coord.en.a.da. ra.dial. a.dmensinnal. a.ssoria.d.a à inter fa.ce

$\hat{\mu}=\left(\frac{\mu_{2}}{\mu_{1}}\right)-$ Razão das viscosidades

$u_{1}$ - Velocidade do meio 1

$u_{2}-$ Velocidade do meio 2

$u^{*}-$ Velocidade do fluido interno

$\bar{u}-$ Velocidade média

$\bar{u}^{*}$ - Velocidade média do fluido interno

$\bar{u}_{1}$ - Velocidade do meio 1

$\bar{u}_{2}-$ Velocidade do meio 2

w-Potência de bombeamento

L-Comprimento do duto ou placa

$Q-$ Vazẫo volumétrica

$Q^{*}$ - Vazão volumétrica do escoamento do fluido interno em contato com a parede

$Q_{1}-$ Vazão voiumétrica do meto 1

$Q_{2}-$ Vazão volumétrica do meio 2

$\bar{\tau}_{n}-$ Tensão viscosa

$\bar{f}-$ Fator de atrito médio

$f_{i}-$ Fator de atrito interno

$\rho-$ Densidade

$\rho_{1}-$ Densidade associada ao meio 1

$D_{i n}-$ Diâmetro hidraulico

$D h_{1}$ - Diâmetro hidraulico interno

C-Constante

$\bar{v}-$ Viscosidade

$\overline{R e}-$ Núumero de Reynolds

$R e_{i}-$ Número de Reynolds interno

$\frac{d v}{d r}-$ Gradiente de velocidade 


\section{Objetivo}

Este trabalho visa estudar o comportamento de fluidos newtonianos imiscíveis, em condições de escoamento em regime laminar anular horizontal completamente desenvolvido (água-óleo), variando-se a temperatura de mistura, e considerando-se a consequente variação da relação de viscosidades. As grandezas de interesse, na análise efetuada, são: perfis de velocidade, gradientes de pressão, vazões mássicas totais constantes e vazão mássica do fluido interno constante, e as consequências na variação da potência de bombeamento.

\section{Desenvolvimento}

O estudo de sistemas água-óleo é de fundamental importância para diversos processos industriais. Procedimentos como a recuperação secundária de petróleo e o bombeamento de óleos de viscosidade elevada- mediante a injeção conjunta de água- exemplificam situações na indústria petrolífera em que o comportamento da interface água-óleo traz importantes consequências para a eficiência da produção, sob o ponto de vista energético [Angeli, P., \& Hewitt, G. F. (2000); Brauner, N., (2001); E. Nogueira, R. M. Cotta (1988); Hasson, D. Orell, A., \& Fink, M. (1974); Vanegas Prada, J. W., \& Bannwart, A. C. (1999)]. A água e o óleo, quando submetidos a processo de escoamento, formam um sistema heterogêneo composto por duas fases que apresenta entre elas uma fronteira chamada de interface, que é a superfície de separação entre as duas fases. A espessura da interface deve ser bem definida atingindo espessuras de poucos milímetros ou mesmo mícron, sendo que a disposição dos fluídos depende do tipo de escoamento. $\mathrm{O}$ fato de o petróleo ser, de um modo geral, mais viscoso e menos denso que a água faz com que, no escoamento simultâneo desses dois fluidos, a água apresente a tendência de se deslocar com maior velocidade em relação ao óleo. As propriedades dos fluídos (densidade, viscosi- dade e tensão superficial) e fenômenos físicos (temperatura, capilaridade, força gravitacional e velocidade de injeção do fluído) influenciam diretamente na resposta do sistema. Com isso, constata-se que a queda na pressão e a redução na potência de bombeamento, em regime laminar, são maiores do que em condições de escoamento turbulento-turbulento. Além disso, para um núcleo altamente viscoso, o regime, no núcleo, é essencialmente laminar, evidenciando que o estudo de um escoamento bifásico líquido-líquido em regime laminar é muito importante tanto em termos teóricos quanto práticos. Além disso, há um significativo aumento da taxa de transferência de calor quando uma camada de fluido de maior condutividade é inserida no escoamento, na forma de um fino filme próximo da parede do conduto [Bentwich, M. \& Sideman, S. (1964); E. Nogueira, L. B. Dantas, R. M. Cotta (2002);E. Nogueira, R. M. Cotta (1990)].

O presente trabalho é uma extensão dos resultados apresentados por Nogueira (1988), com relação ao escoamento em regime laminar horizontal de fluidos imiscíveis. Nele se explora a variação da temperatura de mistura dos meios e sua influência na relação de viscosidade, água-óleo e as consequentes variações em parâmetros associados ao regime laminar horizontal de fluidos imiscíveis em escoamento anular. É de grande interesse, teórico e prático, a obtenção da espessura do fluido externo para a condição de mínima potência de bombeamento, uma vez que esta espessura afeta significativamente a taxa de transferência de calor entre os fluidos e a parede do duto. Deve haver, evidentemente, uma relação de compromisso entre a mínima potência de bombeamento e a máxima transferência de calor para o regime de escoamento em análise.

O escoamento laminar desenvolvido de fluidos Newtonianos imiscíveis em um duto (Figura 1), sem estratificação e com propriedades constantes, é analiticamente descrito pela seguinte forma já simplificada da equação de quantidade de movimento na direção axial [Nogueira (1988)]: 


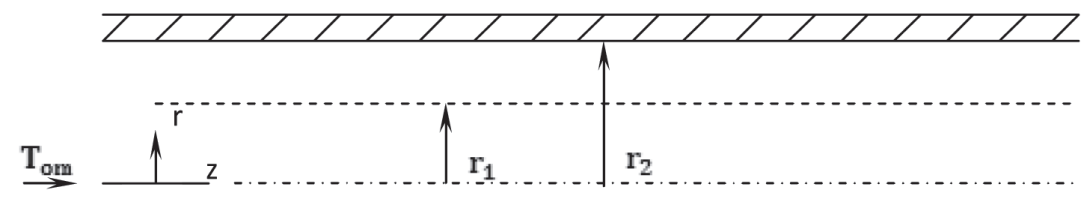

(r)

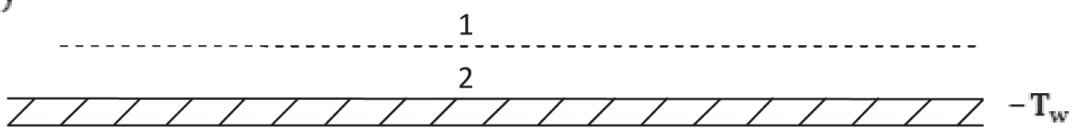

Figura 1: Representação esquemática de escoamento anular de fluidos imiscíveis

$\frac{\mu_{m}}{r^{n}} \frac{d}{d r}\left[r^{n} \frac{d u_{m}}{d r}\right]=-k \equiv \frac{d p}{d z}$

Eq. 1

$r_{m-1}<r<r_{m}, m=1,2$

$n=0$ (Placas Planas Paralelas)e $n=1$ (Duto de Seção Circular)

Definindo-se

$R=\frac{r}{r_{2}}, \quad \delta=\frac{r_{1}}{r_{2}}, \quad \hat{\mu}=\frac{\mu_{2}}{\mu_{1}}$

Para se obter os campos de velocidades é utilizada a Eq. 1 e as seguintes condições de contorno:

$$
\begin{gathered}
\frac{d u_{1}}{d r}=0, \quad r=r_{0} \\
u_{1}=u_{2}, \quad r=r_{1} \\
\mu_{1} \frac{d u_{1}}{d r}=\mu_{2} \frac{d u_{2}}{d r}, \quad r=r_{1} \\
u_{2}=0, \quad r=r_{2}
\end{gathered}
$$

Levando-se aos seguintes perfis de velocidades nos meios 1 e 2:

$$
\begin{aligned}
& u_{1}=\frac{K r_{2}{ }^{2}}{2 \mu_{2}(n+1)}\left[-\delta^{2}+1+\hat{\mu}\left(\delta^{2}-R^{2}\right)\right] \\
& u_{2}=\frac{K r_{2}{ }^{2}}{2 \mu_{2}(n+1)}\left[1-R^{2}\right]
\end{aligned}
$$

A velocidade média total no canal é definida como:

$$
\bar{u}=\frac{\sum_{m=1}^{2} \int_{r_{m-1}}^{r_{m}} r^{n} u_{m}(r) d r}{\int_{r_{0}}^{r_{2}} r^{n} d r}
$$

Tal que:

$\bar{u}=\frac{K r_{2}{ }^{2}}{2 \mu_{2}(n+1)}\left\{\frac{2}{n+3}\left[\delta^{n+3}(\hat{\mu}-1)+1\right]\right\}$ 
Para a determinação das velocidades médias dos fluídos 1 e 2 são utilizadas as seguintes expressões, de forma similar ao efetuado na Eq. 4:

$\bar{u}_{1}=\frac{K r_{2}{ }^{2}}{2 \mu_{2}(n+1)(n+3)}\left\{(n+3)\left[1+\delta^{2}(\hat{\mu}-1)\right]-(n+1) \hat{\mu} \delta^{2}\right\}$

Eq. 6

$\bar{u}_{2}=\frac{K r_{2}{ }^{2}}{2 \mu_{2}(n+1)(n+3)}\left\{\frac{(n+3)\left(1-\delta^{n+1}\right)+(n+1)\left(\delta^{n+3}-1\right)}{1-\delta^{n+1}}\right\}$

Eq. 7

O perfil de velocidade para escoamento unidimensional, e grandezas relacionadas é facilmente deduzido das equações anteriores:

$u^{*}=\frac{K^{*} r_{2}^{2}}{2 \mu_{1}(n+1)}\left[1-R^{2}\right]$

Eq. 8

A potência de bombeamento é obtida através da seguinte expressão:

$w=K L Q$

Eq. 9

Onde a vazão volumétrica total é obtida através da soma das vazões individuais.

$Q=Q_{1}+Q_{2}$

Eq. 10

Grandezas similares para escoamento unidimensional são obtidas das equações desenvolvidas tomando-se $\delta=0$ ou $\delta=1$ :

$Q^{*}=\frac{K^{*} r_{2}{ }^{2} A}{\mu_{1}(n+1)(n+3)}$

$w^{*}=K^{*} L Q^{*}$

Para condição de trabalho de vazões volumétricas interna e total, $\left(\mathrm{Q}_{1} \mathrm{e} \mathrm{Q}^{*}\right)$, iguais, temos:

$\bar{u}_{1} A=\bar{u}^{*} A$

$\frac{K}{K^{*}}=\frac{2 \hat{\mu}}{\delta^{n+1}\left\{(n+3)\left[1+\delta^{2}(\hat{\mu}-1)\right]-(n+1) \hat{\mu} \delta^{2}\right\}}$

$\frac{w}{w^{*}}=\frac{K}{K^{*}}\left[1+\frac{(n+3)\left(1-\delta^{n+1}\right)+(n+1)\left(\delta^{n+3}-1\right)}{\delta^{n+1}\left\{(n+3)\left[1+\delta^{2}(\hat{\mu}-1)\right]-(n+1) \hat{\mu} \delta^{2}\right\}}\right]$

Considerando-se condições de trabalho Gradientes de pressão (K e $\left.\mathrm{K}^{*}\right)$ iguais, temos as seguintes relações:

$\frac{w}{w^{*}}=\frac{Q}{Q^{*}}$

$\frac{w}{w^{*}}=\delta^{n+1} \frac{u_{1}}{\bar{u}^{*}}\left(1-\delta^{n+1}\right) \frac{u_{2}}{\bar{u}^{*}}$

$\frac{\bar{u}_{1}}{\bar{u}^{*}}=\frac{(n+3)\left[1+\delta^{2}(\hat{\mu}-1)\right]-(n+1) \hat{\mu} \delta^{2}}{2 \hat{\mu}}$

$\frac{\bar{u}_{2}}{\bar{u}^{*}}=\frac{(n+3)\left(1-\delta^{n+1}\right)-(n+1)\left(\delta^{n+3}-1\right)}{2 \hat{\mu} \delta^{n+1}}$

Eq. 19 
$\frac{w}{w^{*}}=\frac{K}{K^{*}}$

$\frac{K}{K^{*}}=\frac{\hat{\mu}}{\delta^{n+3}(\hat{\mu}-1)+1}$

Fator de atrito na parede é definido por:

$\bar{\tau}_{n}=-\bar{f} \frac{\rho \bar{u}^{2}}{2 D_{h}}$

Tal que:

$\bar{f}=\frac{2 K D_{h}}{\rho \bar{u}^{2}}$

A partir da equação da velocidade média (Eq. 5), estipulando-se uma constante $C=\frac{n+3}{2}$, encontrou-se uma expressão para o gradiente de pressão $(\mathrm{K})$ :

$K=\frac{2 \bar{u} \mu_{2}(n+1) C}{r_{2}^{2}\left[\delta^{n+3}(\hat{\mu}-1)+1\right]}$

Eq. 24

Substituindo-se o gradiente de pressão na equação do fator de atrito (Eq. 30) tem-se:

$\bar{f}=\frac{2 \bar{u} \mu_{2}(n+1) C 2 D_{h}}{r_{2}^{2}\left[\delta^{n+3}(\hat{\mu}-1)+1\right] \rho \bar{u}^{2}}$

Sabendo-se que:

$$
\begin{aligned}
\bar{\rho} & =\frac{\bar{\mu}}{\bar{v}} \\
\overline{R e} & =\frac{\bar{u} D_{h}}{\bar{v}} \\
D h & =2(2-n) r_{2} \\
D h_{1} & =\delta D h
\end{aligned}
$$

Encontra-se a seguinte expressão para o fator de atrito:

$\bar{f} \cdot \overline{R e}=\frac{8(n+1)(n+3)(2-n)^{2}}{\left[\delta^{n+3}\left(\frac{1}{\hat{\mu}}-1\right)+1\right]\left[\delta^{n+3}(\hat{\mu}-1)+1\right]}$

De forma similar defini-se o fator de atrito interfacial como:

$$
\begin{aligned}
& \tau_{i}=-\left.\mu \frac{d v}{d r}\right|_{r=\delta} \\
& f_{i}=\frac{2 K D h_{1}}{\rho_{1} \bar{u}_{1}{ }^{2}}
\end{aligned}
$$

Considerando a condição de trabalho como sendo vazão mássica interna constante obteve-se:

$$
f_{i} \operatorname{Re}_{i}=\frac{16 \hat{\mu}(n+1)(n+3) \delta(2-n)^{2}}{(n+3)\left[1+\delta^{2}(\hat{\mu}-1)\right]-(n+1) \hat{\mu} \delta^{2}}
$$




\section{Resultados gráficos}

\subsection{Duto Circular $(n=1)$}

No sistema água-óleo para duto circular (Figura 2), quando se mantém constante a vazão mássica total, percebe-se que, à medida que se adiciona o fluido menos viscoso na parede, a potência de bombeamento vai diminuindo até alcançar o valor da relação entre as viscosidades $\widehat{\mu}=0.168$, função da temperatura de trabalho.

Para gradiente de pressão constante, em certo comprimento de duto, nota se que, à medida que se adiciona o fluido menos viscoso na parede, a potência de bombeamento aumenta até o inverso da relação entre as viscosidades.

No caso em que se mantém constante a vazão do fluido interno, ocorre uma queda na potência de bombeamento quando se adiciona o fluido menos viscoso na parede. Observase que, à medida que se vai reduzindo a área disponível para o escoamento, a potência de bombeamento cresce, após passagem por um valor mínimo, de forma que, no limite, quando a área de escoamento tende a zero, esta razão tende ao infinito.

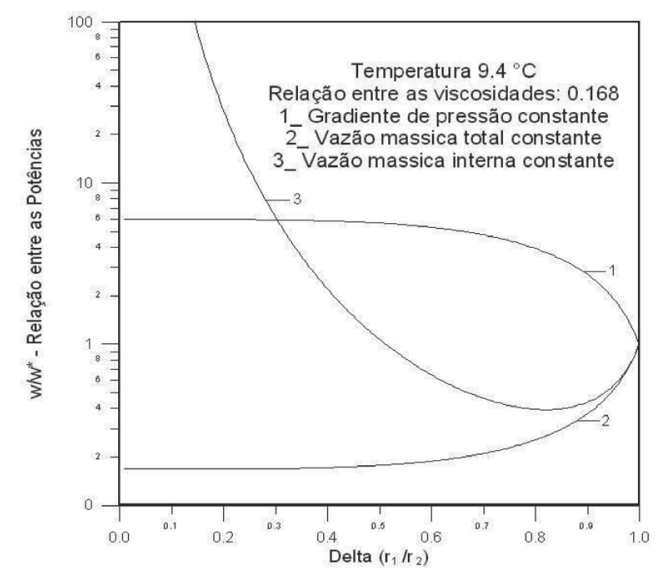

Figura 2- Relação entre as potências de bombeamento à medida que se adiciona água entre o óleo e a parede

Efetuou-se uma análise, (Figura 3), a partir da condição de trabalho: vazão mássica do fluído interno constante. Como a relação entre as viscosidades depende da temperatura, efetuou-se uma análise para a potência de bombeamento para diferentes valores de temperatura; condição em que foi efetuado o levantamento e cálculo das diferentes relações entre as viscosidades. Observou-se que, em todos os casos, qualquer que seja a temperatura, ocorre ponto de mínimo para o valor da espessura do fluido externo. Essa espessura mínima, para uma dada temperatura, é, em tese, o valor mais adequado para a espessura do filme de água entre o óleo e a parede do duto, com relação à potência de bombeamento. $\mathrm{O}$ aspecto mais interessante e expressivo dessa análise, observado através dos resultados da Figura 3 , é que há um ponto de inflexão para a temperatura, com relação ao ponto de mínimo na potência de bombeamento, ou seja, a potência de bombeamento tem, também, um ponto mínimo com relação à variação da temperatura (Figura 4).

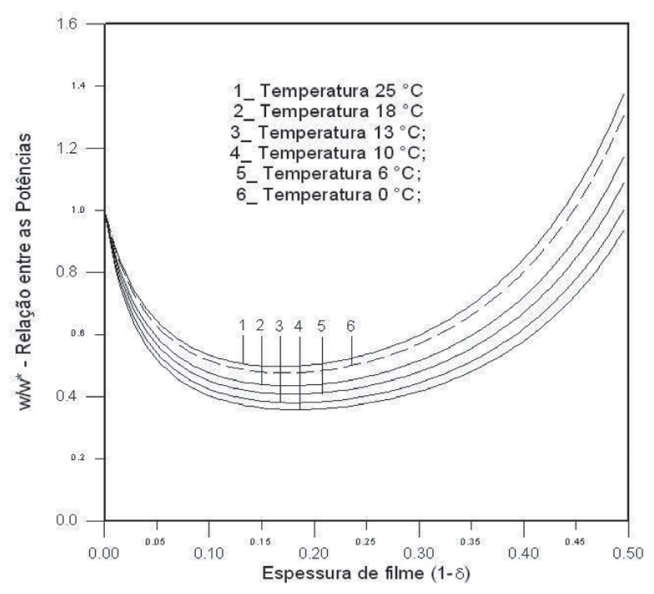

Figura 3 - Relação entre as potências de bombeamento em função da relação entre as viscosidades (água/óleo), considerando-se a fração de água entre o óleo e a parede do duto (Espessura de filme)

Portanto, a partir da Figura 3, foi efetuado o cálculo dos valores de mínima potência em função da temperatura, gerando-se a (Figura 4). Demonstra-se que a relação entre as potências é mínima próxima da temperatura de $9.4^{\circ}$ C. Há, neste caso, uma redução de aproximadamente $64 \%$ na potência de bombeamento.

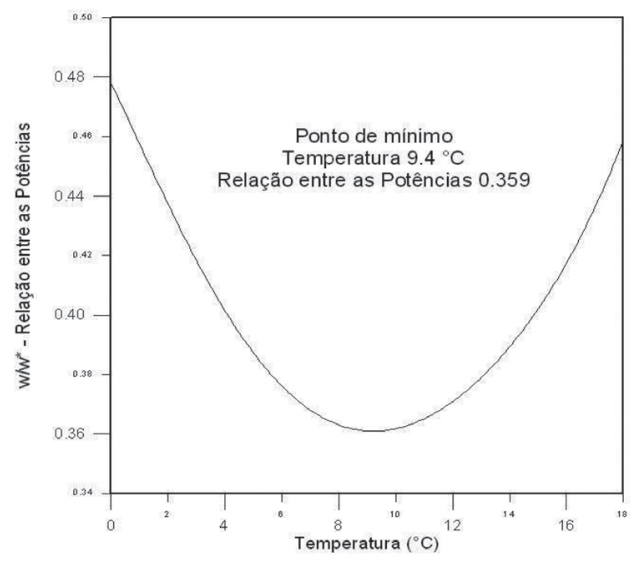

Figura 4 -Relação entre as potências de bombeamento em função da temperatura (o ponto de mínimo entre as potências de bombeamento ocorre à temperatura de $9.4^{\circ} \mathrm{C}$ )

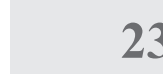

\section{.}
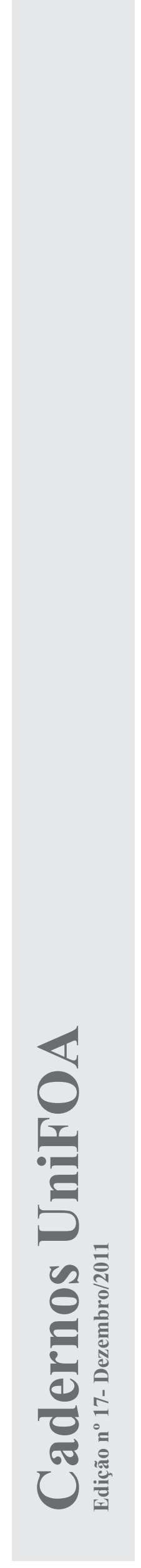

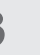


Efetuou-se um estudo do fator de atrito $(\bar{f} \cdot \overline{R e})$ na mínimo para cada temperatura. Como esperado, para delta igual a zero ou delta igual a um, escoamento unifásico, temos parede do duto circular ao longo de delta (Figura 5), para diferentes valores de temperatura, onde se percebe que existe um ponto de o valor clássico da literatura ( $f . R e=64)$. Os resultados, neste caso, refletem os valores qualitativos obtidos para a potência de bombeamento e demonstram a consistência do modelo desenvolvido para duto de seção reta circular.

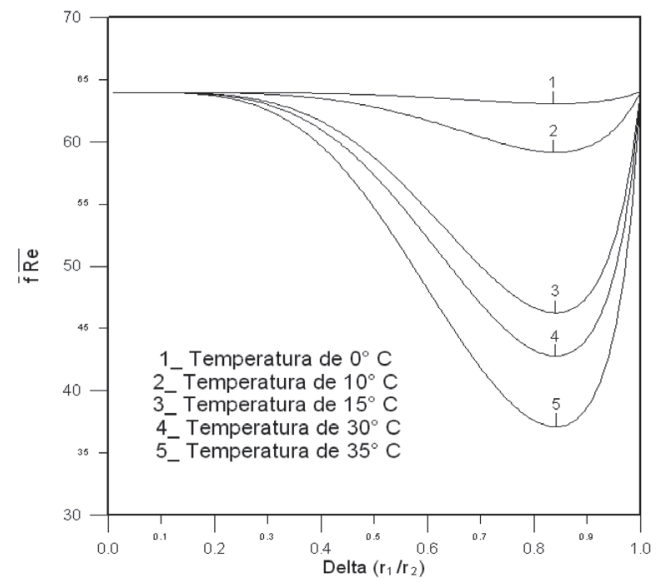

Figura 5 - Fator de atrito em função da fração do óleo no escoamento $\left(\delta=\frac{r_{1}}{r_{2}}\right)$

$\mathrm{Na}$ (Figura 6), estudamos o fator de atrito na interface dos fluidos para diferentes valores de temperaturas. $\mathrm{O}$ valor do fator de atrito vai diminuindo conforme a temperatura diminui até atingir o ponto mínimo que é de $9.4^{\circ} \mathrm{C}$. A partir deste valor, como esperado, volta a subir conforme vai ocorrendo a queda de temperatura. Para valor de delta igual a zero o fator de atrito é zero, em função da derivada da velocidade ser igual a zero no centro da tubulação. Para delta igual a 1.0 (um) temos o valor clássico do escoamento unifásico $(f . R e=64)$, o que corrobora para a consistência do modelo.

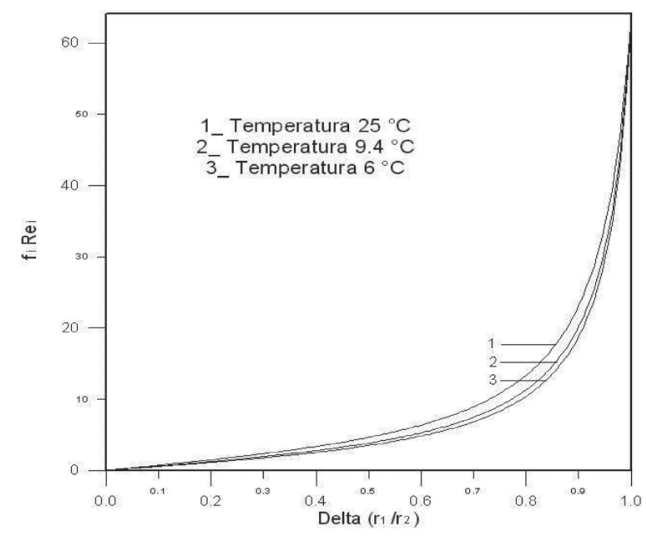

Figura 6 - Fator de atrito interfacial em relação à fração de óleo no escoamento $\left(\delta=\frac{r_{1}}{r_{2}}\right)$

\subsection{Placas planas paralelas $(n=0)$}

No sistema água-óleo para placas planas paralelas (Figura 7), quando se mantém constante a vazão mássica total, percebe-se que, à medida que se adiciona o fluido menos viscoso na parede, a potência de bombeamento vai diminuindo até alcançar o valor da relação $\widehat{\mu}$ $=0.365$, função da temperatura de trabalho.

Para gradiente de pressão constante, em certo comprimento de duto, nota se que, à medida que se adiciona o fluido menos viscoso na parede, a potência de bombeamento aumenta até o inverso da relação entre as viscosidades. Comportamento também observado para a geometria de duto circular.

No caso em que se mantém constante a vazão do fluido interno, ocorre uma queda na potência de bombeamento quando se adiciona o fluido menos viscoso na parede. Observa-se, ainda, que, à medida que se vai reduzindo a área disponível para o escoamento, a potência de bombeamento cresce, após passagem por um valor mínimo, de forma que, no limite, quando a área de escoamento tende a zero, esta razão tende ao infinito.

Esses resultados, em termos qualitativos, já eram esperados, em função dos resultados obtidos para duto de seção reta circular.

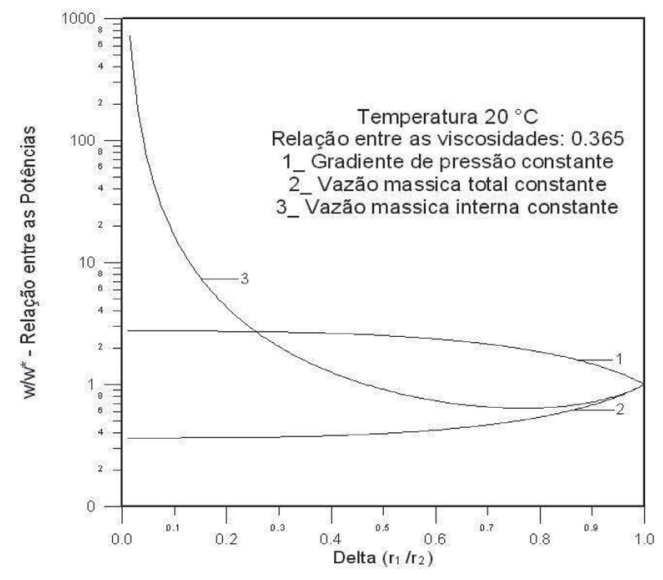

Figura 7- Relação entre as potências de bombeamento à medida que se adiciona água entre o óleo e a parede

Demonstra-se, na (Figura 8), que, quando se utiliza a condição de trabalho de vazão mássica interna constante, para diferentes temperaturas, temos diferentes pontos de mínimo(delta), onde se tem a maior redução de potência de bombeamento. Determinou-se o ponto de mínimo, $\left(\delta^{\prime}=\frac{r_{1}}{r_{2}}\right)$, para diferentes temperaturas (Figura 9). 


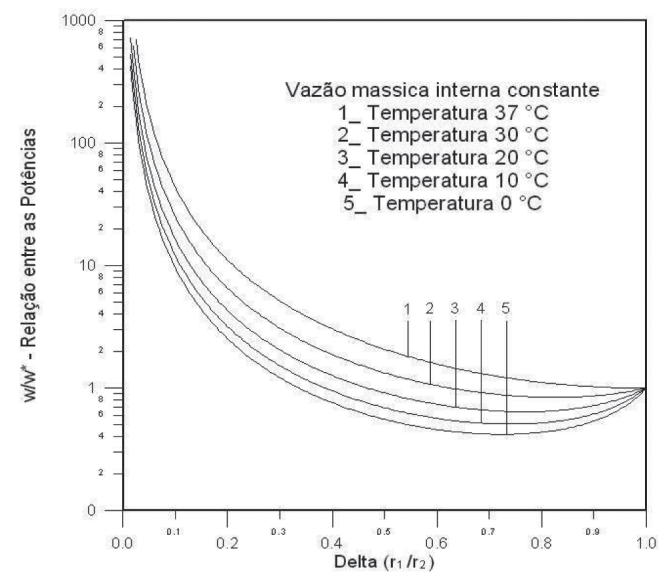

Figura 8- Relação entre as potências em função da fração de óleo no escoamento, para diferentes temperaturas.

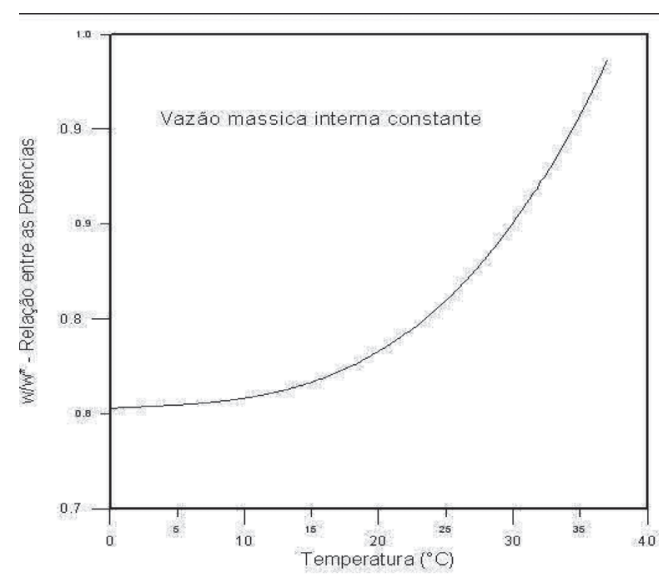

Figura 9 - Pontos de mínimo para fração de óleo no escoamento, em função da temperatura.

Efetuou-se um estudo para o fator de atrito na parede, para diferentes temperaturas de trabalho (Figura 10). Observou-se que existe um ponto mínimo para cada temperatura, e que para delta igual a zero (0.0) ou igual a um (1.0) obteve-se, novamente, como esperado, o valor de fator de atrito para escoamento unifá$\operatorname{sico}(\mathrm{f} . \mathrm{Re}=96)$.

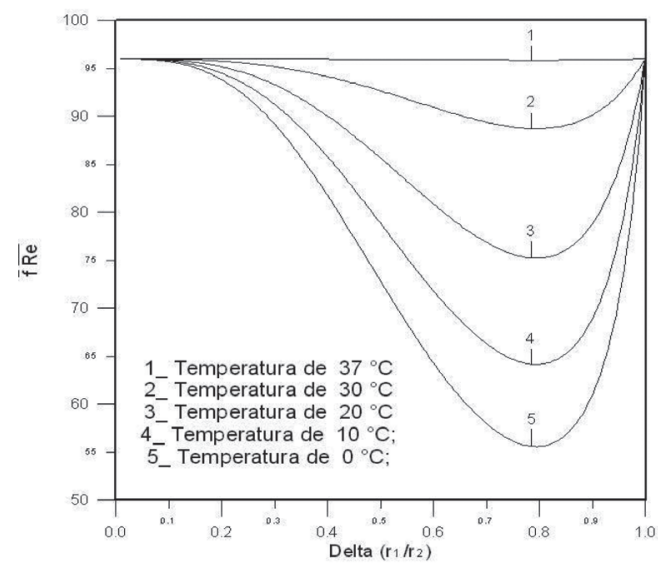

Figura 10 - Fator de Atrito em função da fração de óleo no escoamento $\left(\delta=\frac{r_{1}}{r_{2}}\right)$
$\mathrm{Na}$ (Figura 11), analisou-se o fator de atrito na interface dos fluidos para diferentes valores de temperaturas. O valor do fator de atrito vai diminuindo conforme a temperatura diminui. O comportamento qualitativo é similar ao já observado para duto circular. Para valores de delta igual a zero ou delta igual a um, o resultado numérico coincide com unifásico para placas planas paralelas (f.Re $=96$ ). Observa-se que acima de $36^{\circ} \mathrm{C}$, em placas planas paralelas, não há nenhuma vantagem na utilização de água como filme, pois não há redução na potência de bombeamento.

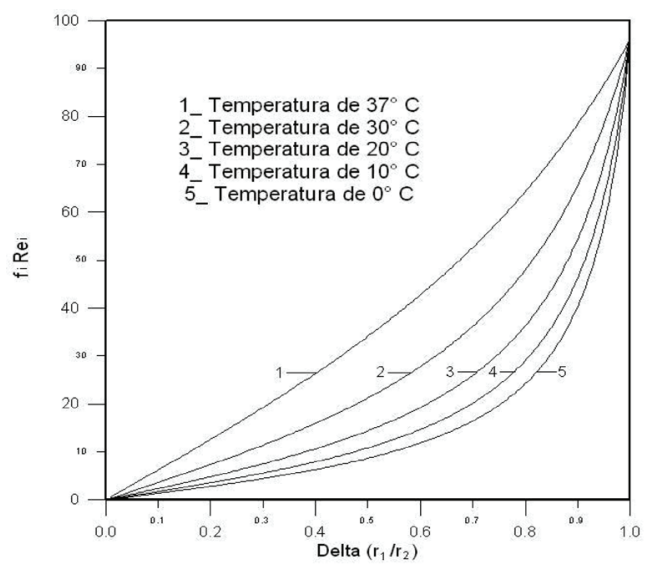

Figura 11 - Fator de Atrito interfacial em função da fração de óleo no escoamento $\left(\delta=\frac{r_{1}}{r_{2}}\right)$.

\section{Conclusão}

Todos os resultados qualitativos e quantitativos encontrados neste trabalho estão compatíveis com o esperado fisicamente e demonstram que o modelo desenvolvido tende para os resultados clássicos da literatura para escoamento unifásico.

A conclusão mais relevante do trabalho encontra-se no fato de que há, para escoamento em seção reta circular, uma temperatura onde a relação entre as potências de bombeamento é mais vantajosa. A temperatura, neste caso, é $9.4{ }^{\circ} \mathrm{C}$, e a redução na potência de bombeamento é de aproximadamente $64 \%$.

Outro ponto relevante na análise efetuada está relacionado com o fato de que há uma temperatura limitante para o uso de água como filme, para a redução da potência de bombeamento, em escoamento entre placas planas paralelas. Está temperatura limite, para sistema água-óleo, é aproximadamente igual a $36{ }^{\circ} \mathrm{C}$.

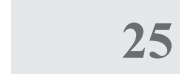

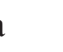
政 
As situações de um mínimo valor de temperatura adequada para o melhor desempenho hidrodinâmico em dutos circulares, e uma máxima temperatura em que é desvantajoso utilizar o escoamento anular para placas planas paralelas,

\section{Referências Bibliográficas}

1. Angeli, P., \& Hewitt, G. F. (2000), Structure in horizontal oil-water flow. Int. J. Multiphase Flow, 26, 1117-1140.

2. Brauner, N., (2001), The prediction of dispersed flows in liquid-liquid and gásliquid sistems. Int. J. Multiphase Flow, 27, 59-76

3. E. Nogueira (1988). Escoamento laminar e transferência de calor em fluidos imiscíveis sem estratificação. Tese de Mestrado. Instituto Tecnológico de Aeronáutica - ITA

4. Hasson, D. Orell, A., \& Fink, M. (1974). A study of vertical annular liquid-liquid flow - Part I: Laminar condictions, Paper No. 5, Multiphase Flow Systems Symp., Inst. Chem Engng. Symp., Ser. No. 38, 1-15.

5. Vanegas Prada, J. W., \& Bannwart, A. C. (1999). Pressure drop in vertical core annular flow, XV COBEM, Congresso Brasileiro de Engenharia Mecânica, Águas de Lindoia, SP, CD-ROM. são absolutamente inusitadas e demandam uma análise de transferência de calor para as situações críticas, com o objetivo de se obter uma relação custo/benefício entre a potência de bombeamento e o processo de transferência de calor.

6. Vanegas Prada, J. W., \& Bannwart, A. C. (1999). Pressure drop in vertical core annular flow, XV COBEM, Congresso Brasileiro de Engenharia Mecânica, Águas de Lindoia, SP, CD-ROM.

7. Bentwich, M. \& Sideman, S. (1964). Temperature distribuition and heat transfer in

8. E. Nogueira,L. B. Dantas, R. M. Cotta. (2002). "Thermohyfraulic Performance in Cocurrent Laminar Flow of Immiscibible Liquids". II Encontro de Ciências Térmicas - ENCIT, 12/88, pp. 307-310, Águas de Lindoia - S.P

9. E. Nogueira, R. M. Cotta. "Heat Transfer Solution in Laminar Cocurent of Immiscibible Liquids". Warme-Ünd Stoffübertagung, Vol. 25, pp. 361-367, Springer-Verlag 1990 annular two-phase (liquid-liquid) flow, Canad. J. Chem. Eng., 9-13.

\author{
Endereço para Correspondência: \\ Artur Kimura \\ artur_kimura@hotmail.com \\ Centro Universitário de Volta Redonda - UniFOA \\ Campus Universitário Olezio Galotti \\ Av. Paulo Erley Abrantes, 1325 \\ Três Poços - Volta Redonda - RJ \\ CEP: $27.240-560$
}

Informacões bibliográficas:

Conforme a NBR 6023.2002 da Associaç̃o Brasileira de Normas Técnicas (ABNT), este texto científico publicado em periódico eletrônico deve ser citado da seguinte forma: KIMURA, Artur; OLIVEIRA, Carolina Valente de; NOGUEIRA, Elcio. Hidrodinâmica de Líquidos Imiscíveis (Água-Óleo) em escoamentos internos: Seção Reta Circular e Placas Planas Paralelas. Cadernos UniFOA. Volta Redonda, Ano VI, n. 17, dezembro 2011. Disponível em: < http://www.unifoa.edu.br/cadernos/edicao/17/17.pdf> 